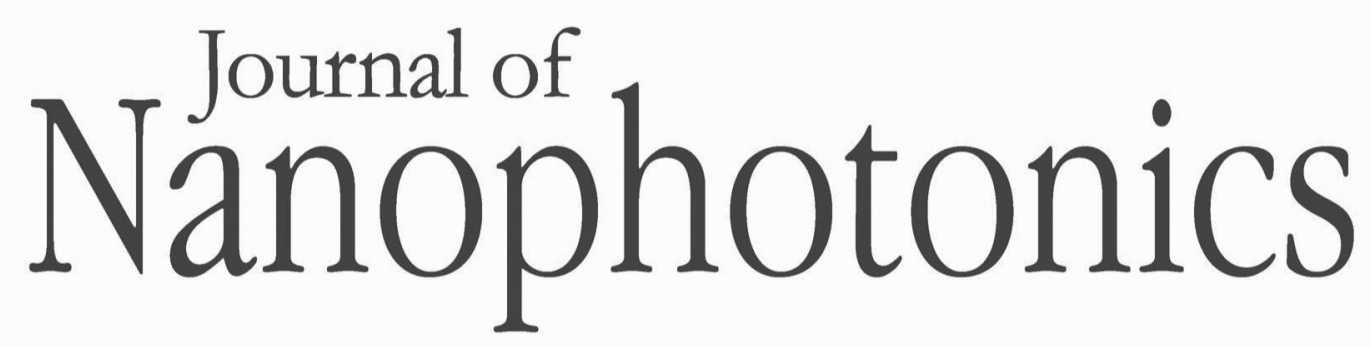

SPIEDigitalLibrary.org/jnp

\title{
Effect of size of aluminum/silicon dioxide/aluminum nanosandwich films on their optical properties
}

Yi-Jun Jen

Hung-Sheng Liao

Meng-Jie Lin 


\title{
Effect of size of aluminum/silicon dioxide/aluminum nanosandwich films on their optical properties
}

\author{
Yi-Jun Jen,* Hung-Sheng Liao, and Meng-Jie Lin \\ National Taipei University of Technology, Department of Electro-Optical Engineering, \\ No. 1, Sec. 3, Chung-Hsiao E. Road Taipei 106, Taiwan
}

\begin{abstract}
Three aluminum (Al)/silicon dioxide $\left(\mathrm{SiO}_{2}\right)$ /aluminum (Al) nanosandwich films (SWFs) of various heights were fabricated using glancing angle deposition. An SWF comprises a 45-nm thick $\mathrm{SiO}_{2}$ layer sandwiched between two $\mathrm{Al}$ nanopillars. The thicknesses of both top and bottom nanopillars were varied from 187.5 to $217.5 \mathrm{~nm}$. The equivalent constitutive and related parameters of each SWF were obtained from the reflection coefficients and transmission coefficients that were measured using a walk-off interferometer. Both the equivalent permittivity and the equivalent permeability of each SWF turned out to be negative real. Exactly how the height of the Al nanopillars of the double negative SWF affects its low reflectance through destructive interference is also examined using the wave tracing method. Moreover, the localized reversed magnetic field in the $\mathrm{SiO}_{2}$ layer of each SWF was simulated by finite-difference timedomain method to qualitatively interpret the negative real permeability. (C) The Authors. Published by SPIE under a Creative Commons Attribution 3.0 Unported License. Distribution or reproduction of this work in whole or in part requires full attribution of the original publication, including its DOI. [DOI: 10 .1117/1.JNP.8.083994]
\end{abstract}

Keywords: metamaterials; optical properties; thin films.

Paper 13121SSP received Oct. 28, 2013; revised manuscript received Feb. 13, 2014; accepted for publication Feb. 17, 2014; published online Mar. 17, 2014.

\section{Introduction}

With the rapid development of metamaterials in the recent decade, related works have demonstrated the novel optical properties of subwavelength structures of such metamaterials, including the negative refractive index ${ }^{1-3}$-from microwave wavelengths to visible wavelengths. To achieve related applications (e.g., perfect lens and cloaking ${ }^{4}$ ), many recent efforts have attempted to reduce the loss of metamaterials. ${ }^{5,6}$ However, between the discovery of metamaterials and the development of metadevices, the mass production of metamaterials is of priority concern. ${ }^{7}$ In addition to electron-beam lithography, ${ }^{8}$ glancing angle deposition ${ }^{9}$ is a relatively facile means of coating a nanostructured thin film with a negative refractive index at visible wavelengths. By depositing at a glancing angle of $89 \mathrm{deg}$ and deposition rate of $1 \mathrm{~nm} / \mathrm{s}$, a slanted $\mathrm{Ag}$ nanorod array (NRA) with a thickness of $160 \mathrm{~nm}$ exhibits a negative real refractive index. Measurement results indicate that the polarization-dependent refractive index is positive real for s-polarization (i.e., the electric field of the incident wave oscillates normally to the rods) and negative real for p-polarization (i.e., the electric field of the incident wave oscillates along the rods). The p-polarized refractive index is $-0.581+i 0.213,-0.307+i 1.621$, and $-0.389+i 1.816$ at wavelengths $\lambda$ of 532, 639, and $690 \mathrm{~nm}$, respectively. ${ }^{10}$ However, the p-polarization transmittance of Ag NRA is only $<5 \%$, owing to its strong light absorption.

A previous work attempted to mitigate the loss problem of metal NRA by fabricating a sandwiched $\mathrm{Al}-\mathrm{SiO}_{2}-\mathrm{Al}$ upright NRA was fabricated through the introduction of rapidly substrate spin during glancing deposition. ${ }^{11}$ This sandwiched NRA exhibits a polarization-independent negative refractive index and equivalent permeability for normal incidence. Additionally,

\footnotetext{
*Address all correspondence to: Yi-Jun Jen, E-mail: jyjun@ntut.edu.tw

This paper is substantially based on the paper titled "Size effect of aluminum/silicon dioxide/aluminum nanosandwich films for negative optical properties," presented at the SPIE Optics+Photonics 2013, NanoScience+Engineering Conference 8818: Nanostructured Thin Films VI, held on August 28-29, 2013, in San Diego, California.
} 
loss of the sandwiched film is sufficiently low to allow light to propagate through and to be reflected back from, the film, subsequently producing interference effect. Antireflection caused by destructive interference from the film is observed and demonstrated herein. The negative phase change caused by backward wave propagation in a negative index medium contributes to the destructive interference between the first two orders of reflected waves.

This work examines how the Al nanopillar thickness affects electromagnetic and optical properties of the $\mathrm{Al}-\mathrm{SiO}_{2}-\mathrm{Al}$ sandwiched. Three samples with $\mathrm{Al}$ nanopillar thicknesses varying from 187.5 to $217.5 \mathrm{~nm}$ are fabricated. Their electromagnetic parameters, including permittivity, permeability, and the bianisotropic parameter, are also derived from optical parameters, including refractive index, forward impedance, and backward impedance. The optical parameters are derived from the reflection and transmission coefficients measured by using walk-off interferometry. Moreover, the low reflectance values for different thicknesses of nanosandwich films (SWFs) are analyzed using the optical parameters. Furthermore, the negative real permeability variation with the size of SWF is investigated through simulation of the localized magnetic field, by using the finite-difference time-domain (FDTD) method. This work reveals that low reflectivity exists over an extended range of thickness of SWF with negative real index. The thicknessdependent optical parameters will be useful in optical thin film design for a novel optical filter that contains both positive and negative index thin films.

\section{Fabrication and Measurement}

In the fabrication of SWF, SWFs were grown by glancing angle deposition (GLAD) technique in an electron-beam evaporation system. The chamber containing the substrates and the targets of aluminum and silicon dioxide was pumped to a base pressure of $4 \times 10^{-6}$ Torr prior to evaporation. The target-to-substrate distance was fixed at $29 \mathrm{~cm}$. The collimated vapor flux of either $\mathrm{Al}$ or $\mathrm{SiO}_{2}$ was directed toward the substrate at an angle of 89 deg to the substrate normal. The substrate spun around its central normal axis at a rate of $10 \mathrm{rpm}$, subsequently causing the growing film to comprise upright nanopillars. A quartz crystal microbalance was placed next to the stage to monitor the deposition rate, which was maintained at approximately $1 \mathrm{~nm} / \mathrm{s}$. Figure 1 presents the top-view and cross-section views of the three SWFs. Table 1 lists the size parameters, including the average diameter and average thickness of each SWF.

Figure 1 describes the system coordinates $(x, y, z)$, as defined for optical measurements, where the $z$-axis is the direction of normal to the surface of the film. According to Fig. 2, the $x(y)$-polarized transmittance $T_{j}$, reflectance $R_{j}$, and absorbance $A_{j}=1-\left(R_{j}+T_{j}\right), j \in\{x, y\}$, of the SWF depends slightly on the orientation of the electric field of normally incident light. Here, the spectra of both transmittance and reflectance are measured on a TRIAX 180 spectrometer (Horiba Jobin Yvon, Opticsvalley, France). The transmittance of all three samples increases with wavelength, and a thicker SWF exhibits lower transmittance. The transmittance of the 420-nm thick SWF increases from 0.312 at $\lambda=400 \mathrm{~nm}$ to 0.687 at $\lambda=700 \mathrm{~m}$, while the transmittance of the 480-nm thick SWF increases from 0.263 at $\lambda=400 \mathrm{~nm}$ to 0.627 at $\lambda=700 \mathrm{~nm}$. Although the thickness of SWF varies from 420 to $480 \mathrm{~nm}$, the reflectance levels of three samples are $<1 \%$.
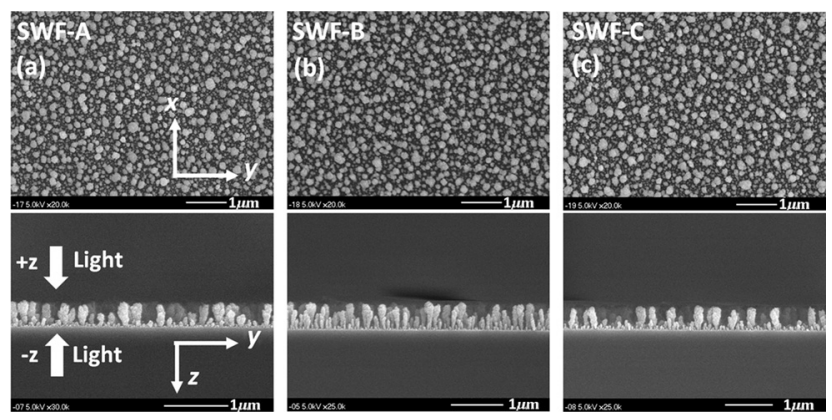

Fig. 1 Top view and cross-sectional scanning electron microscopic images of three $\mathrm{Al}-\mathrm{SiO}_{2}-\mathrm{Al}$ nanosandwich films (SWFs) with total thicknesses of (a) $420 \mathrm{~nm}$, (b) $450 \mathrm{~nm}$, and (c) $480 \mathrm{~nm}$, respectively. 
Table 1 Average diameter and thickness of each sample.

\begin{tabular}{lcccc}
\hline \hline Sample & $\begin{array}{c}\text { Total thickness of } \\
\text { SWF }(\mathrm{nm})\end{array}$ & $\begin{array}{c}\text { Thickness of } \\
\mathrm{SiO}_{2} \text { layer }(\mathrm{nm})\end{array}$ & $\begin{array}{c}\text { Thickness of } \\
\text { Al layer }(\mathrm{nm})\end{array}$ & $\begin{array}{c}\text { Average } \\
\text { diameter }(\mathrm{nm})\end{array}$ \\
\hline SWF-A & 420 & 45.0 & 187.5 & 165.4 \\
SWF-B & 450 & 45.0 & 202.5 & 176.9 \\
SWF-C & 480 & 45.0 & 217.5 & 179.8 \\
\hline \hline
\end{tabular}
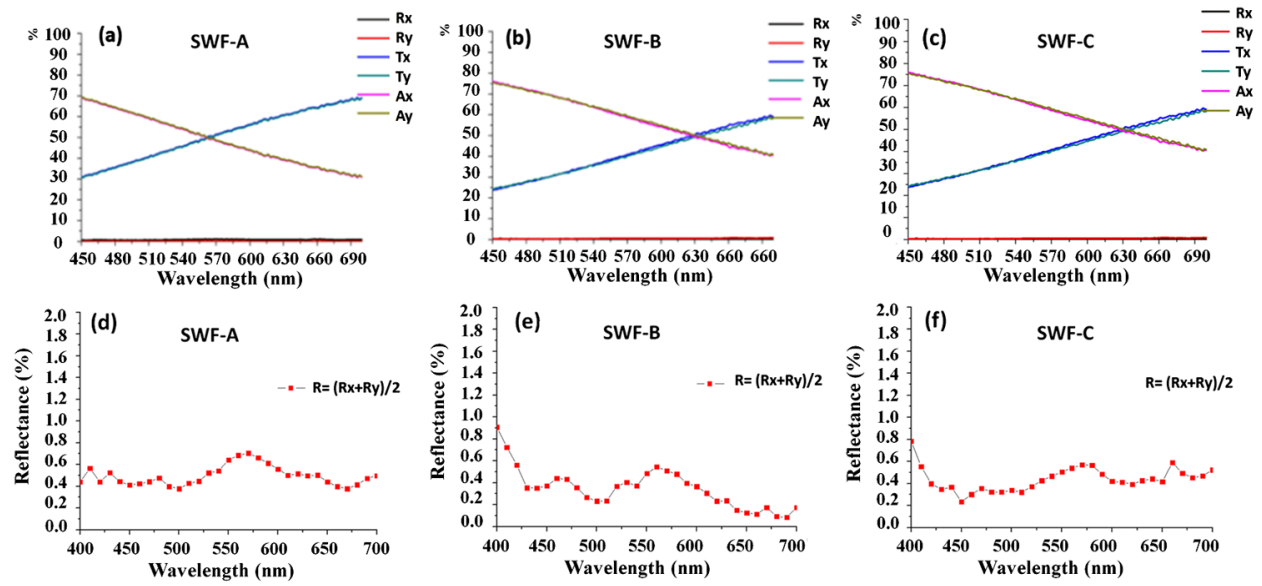

Fig. 2 Measured spectra of the $T_{j}, R_{j}$, and $A_{j}=1-\left(R_{j}+T_{j}\right), j \in\{\mathrm{x}, \mathrm{y}\}$ of (a) SWF-A, (b) SWF-B, and (c) SWF-C and the average reflectance spectra of (d) SWF-A, (e) SWF-B, and (f) SWF-C. Light was normally incident for transmittance measurements, yet obliquely incident at 5 deg to the normal for reflectance measurements.

The equivalent refractive index $N_{j}=N_{j}^{\prime}+i N_{j}^{\prime \prime}$, equivalent relative impedance $Z_{+, j}=Z_{+, j}^{\prime}+i Z_{+, j}^{\prime \prime}$, and $Z_{-, j}=Z_{-, j}^{\prime}+i Z_{-, j}^{\prime \prime}$ are derived from reflection and transmission coefficients measured from both sides of the air/SWF/substrate system using a method described elsewhere. ${ }^{12}$ The equivalent relative impedances $Z_{+, j}=Z_{+, j}^{\prime}+i Z_{+, j}^{\prime \prime}$ and $Z_{-, j}=Z_{-, j}^{\prime}+i Z_{-, j}^{\prime \prime}$ are associated with forward (+) and backward (-) directions of light propagating through the air/SWF/BK7 glass system (Fig. 1). The equivalent relative permittivity $\varepsilon_{j}=\varepsilon_{j}^{\prime}+i \varepsilon_{j}^{\prime \prime}$, equivalent relative permeability $\mu_{j}=\mu_{j}^{\prime}+i \mu_{j}^{\prime \prime}$, and bianisotropic parameter $\xi_{j}=\xi_{j}^{\prime}+i \xi_{j}^{\prime \prime}$ are then calculated from $N_{j}, Z_{+, j}$, and $Z_{-, j}$, respectively. Additionally, the complex-valued reflection coefficients $r_{ \pm, j}$ and transmission coefficients $t_{ \pm, j}$ of the SWF are measured for the SWF deposited on the glass slide, by using a walk-off interferometer with a wavelength-tunable 35 KAP 431-220 Ion Laser System (CVI MellesGriot, Albuquerque). The measurements are also taken at three wavelengths: 476, 568, and $676 \mathrm{~nm}$. Figure 3 plots the values of $N_{j}$ at the three wavelengths and Table 2 lists the values $Z_{+, j}$ and $Z_{-, j}$. Figure 4 presents the equivalent electromagnetic parameters and optical parameters of the three samples. The magnitude of the real part of the refractive index increases with wavelength, subsequently preventing the optical thickness

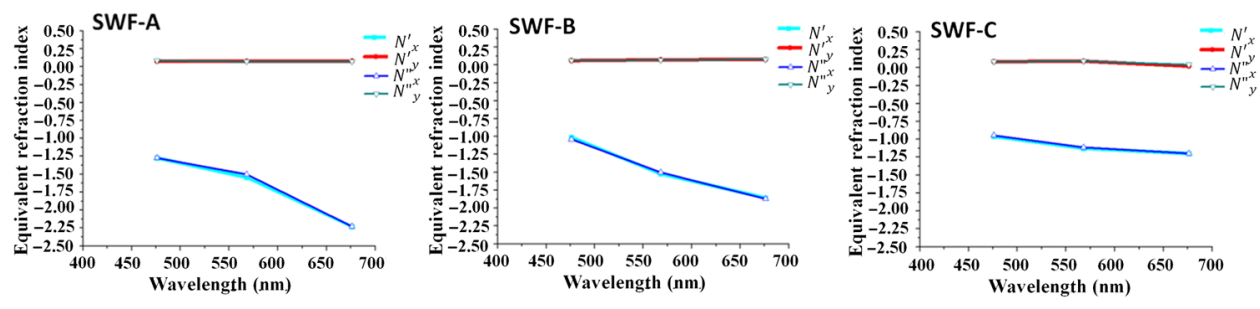

Fig. 3 Measured values of $N_{j}$ for SWF-A, SWF-B, and SWF-C. 
Jen, Liao, and Lin: Effect of size of aluminum/silicon dioxide/aluminum nanosandwich films...

Table $2 Z_{+, j}$ and $Z_{-, j}$ for SWF-A, SWF-B, and SWF-C.

\begin{tabular}{|c|c|c|c|c|c|}
\hline \multirow[b]{2}{*}{ Sample } & \multirow[b]{2}{*}{$\lambda(\mathrm{nm})$} & \multicolumn{2}{|c|}{$Z_{+, j}^{\prime}+i Z_{+, j}^{\prime \prime}$} & \multicolumn{2}{|c|}{$Z_{-, j}^{\prime}+i Z_{-, j}^{\prime \prime}$} \\
\hline & & $x$-polarization & $y$-polarization & $x$-polarization & $y$-polarization \\
\hline \multirow[t]{3}{*}{ SWF-A } & 476 & $0.896-i 0.267$ & $0.929-i 0.194$ & $0.389-i 0.009$ & $0.413-i 0.002$ \\
\hline & 568 & $1.135+i 0.089$ & $1.070+i 0.061$ & $0.638-i 0.233$ & $0.652-i 0.213$ \\
\hline & 676 & $1.278+i 0.260$ & $1.192+i 0.240$ & $0.547-i 0.280$ & $0.549-i 0.282$ \\
\hline \multirow[t]{3}{*}{ SWF-B } & 476 & $0.821-i 0.289$ & $0.857-i 0.202$ & $0.536-i 0.106$ & $0.544-i 0.105$ \\
\hline & 568 & $0.741+i 0.159$ & $0.812+i 0.081$ & $0.483-i 0.087$ & $0.487-i 0.086$ \\
\hline & 676 & $1.119+i 0.108$ & $0.974+i 0.063$ & $0.672-i 0.223$ & $0.682-i 0.211$ \\
\hline \multirow[t]{3}{*}{ SWF-C } & 476 & $1.251+i 0.003$ & $1.304+i 0.052$ & $0.337-i 0.235$ & $0.355-i 0.225$ \\
\hline & 568 & $1.387+i 0.247$ & $1.500+i 0.295$ & $0.318+i 0.149$ & $0.332+i 0.136$ \\
\hline & 676 & $0.905+i 0.164$ & $0.916+i 0.254$ & $0.564+i 0.138$ & $0.570+i 0.140$ \\
\hline
\end{tabular}
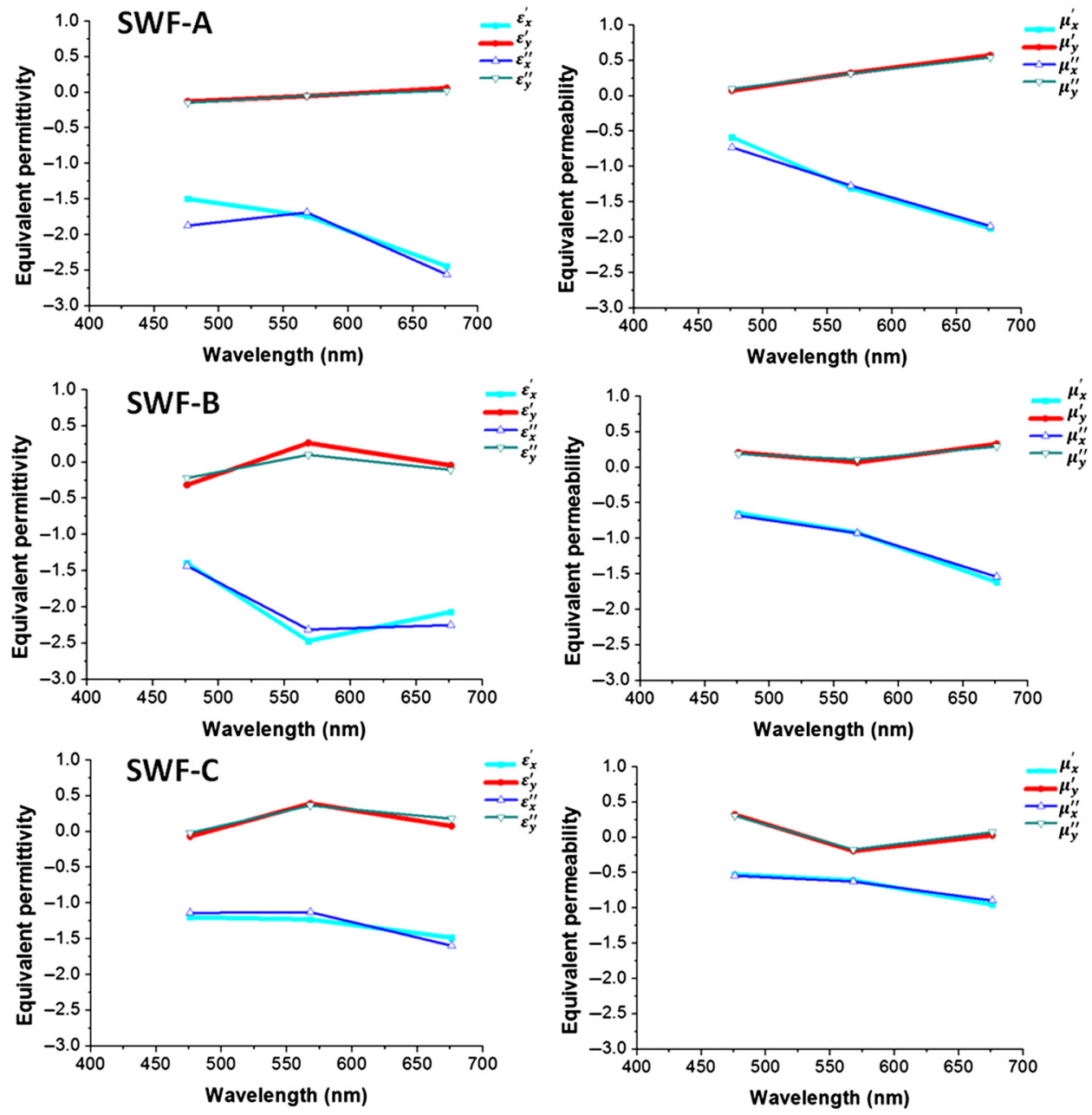

Fig. 4 Measured values of $\varepsilon_{j}$ and $\mu_{j}$ for SWF-A, SWF-B, and SWF-C. 
varying intensively with wavelength. The imaginary part of the refractive index is sufficiently low to have interference between the first two reflected waves from SWF. The value $N_{j}^{\prime \prime}$ is within the range $(0.117,0.137)$ for SWF-A, $(0.046,0.073)$ for SWF-B, and $(0.086,0.098)$ for SWF-C. As the thickness of SWF increases from 420 to $480 \mathrm{~nm}$, the equivalent refractive index is negative; in addition, its average magnitude decreases from -2.256 to -1.132 at a wavelength $\lambda$ of $676 \mathrm{~nm}$. Similarly, the real part of the equivalent permittivity varies from -1.193 to -0.824 as the thickness increases. Both equivalent permittivity and permeability are negative real, and the magnitude of the real part of the permeability increases as wavelength increases.

\section{Wave Tracing}

An attempt is made to exploit the cause of low reflectivity by using the equivalent optical parameters $N_{x}, Z_{+, x}$, and $Z_{-, x}$ to calculate the phase change associated with propagation through SWF, reflection coefficient, and transmission coefficient at both interfaces. The wave propagating through the film is simulated for two cases: the SWF-A at a wavelength of $676 \mathrm{~nm}$ and the SWF-C at a wavelength of $476 \mathrm{~nm}$ (Fig. 5). With incident electric field amplitude of unity, the first-order reflected wave and second-order reflected wave interfere with each other, resulting

\section{SWF-A at a wavelength of $676 \mathrm{~nm}$}

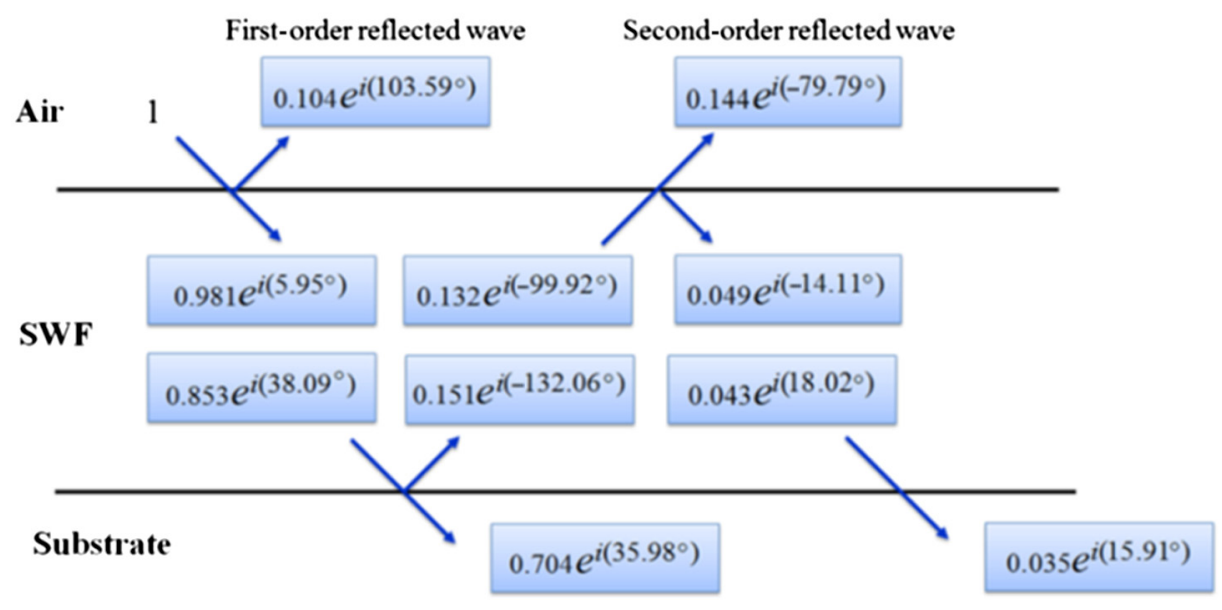

\section{SWF-C at a wavelength of $476 \mathrm{~nm}$}

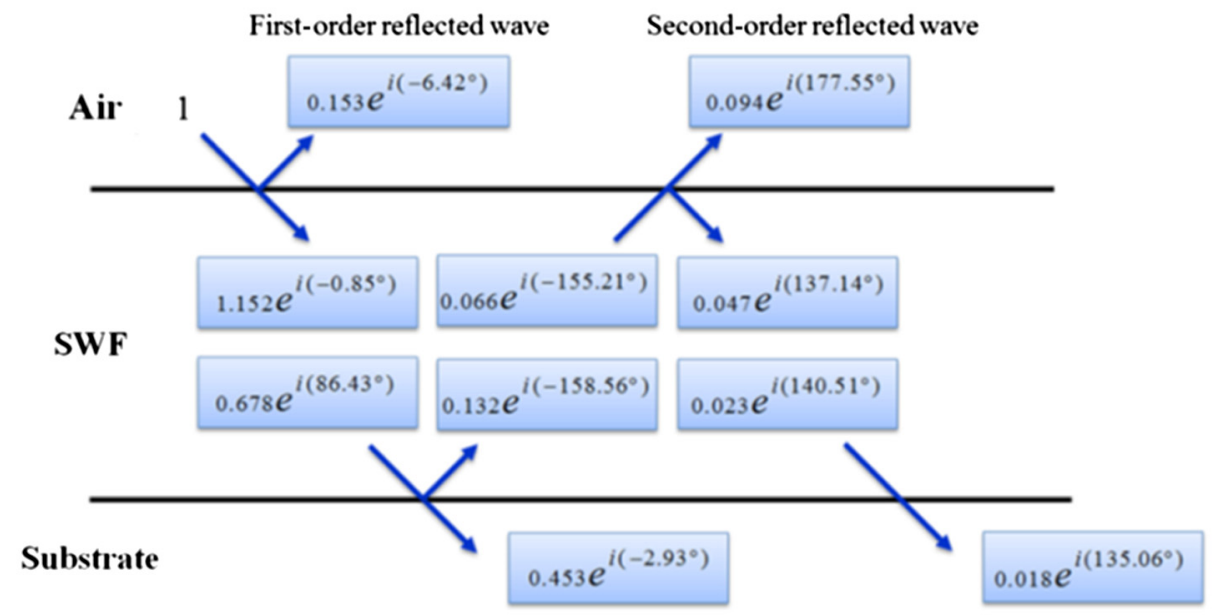

Fig. 5 Wave tracing for SWF normally illuminated by light with an electric field amplitude of unity for SWF-A at a wavelength of $676 \mathrm{~nm}$ and SWF-C at a wavelength of $476 \mathrm{~nm}$. 
Jen, Liao, and Lin: Effect of size of aluminum/silicon dioxide/aluminum nanosandwich films...

in diminished total reflected wave. The total reflected wave becomes $0.104 e^{i\left(103.59^{\circ}\right)}+$ $0.144 e^{i\left(-79.79^{\circ}\right)}=0.041 e^{i\left(88.44^{\circ}\right)}$ for SWF-A at a wavelength of $676 \mathrm{~nm}$ and $0.153 e^{i\left(-6.42^{\circ}\right)}+$ $0.094 e^{i\left(177.55^{\circ}\right)}=0.058 e^{i\left(2.27^{\circ}\right)}$ for SWF-C at a wavelength of $476 \mathrm{~m}$. Both cases perform well in terms of the destructive interference between the first two reflected waves from the SWF, owing to a low loss property of SWF. Owing to the negative real part of the refractive index, the phase associated wave propagation is negative as a result of backward wave propagation. ${ }^{9}$ The near- $\pi$ phase differences between the first-order and second-order reflected waves at wavelengths of 476 and $676 \mathrm{~nm}$ come from the low dispersive optical thickness, as shown in Sec. 2. Although the magnitude of the first-order reflection coefficient of SWF-A than that of SWF-C, the lower loss of SWF-A increases the magnitude of the second-order reflection coefficient of SWF-A more than that of SWF-C. Therefore, both cases have similar destructive interference phenomena.

\section{Magnetic-Field Reversal Analysis}

The negative real part of the permeability arises from the reversal of the magnetic field between a pair of aluminum grains. According to the values in Fig. 4, the average real part of the permeability of SWF-A varies from -0.6684 at $\lambda=476 \mathrm{~nm},-1.2878$ at $\lambda=568 \mathrm{~nm}$ to -1.8588 at $\lambda=676 \mathrm{~nm}$. Additionally, the average real part of the permeability of SWF-B varies from -0.6550 at $\lambda=476 \mathrm{~nm},-0.9240$ at $\lambda=568 \mathrm{~nm}$ to -1.5792 at $\lambda=676 \mathrm{~nm}$. Moreover, the average real part of the permeability of SWF-C varies from -0.5361 at $\lambda=476 \mathrm{~nm}$, -0.6183 at $\lambda=568 \mathrm{~nm}$ to -0.9240 at $\lambda=676 \mathrm{~nm}$. When the thickness of SWF increases from 420 to $450 \mathrm{~nm}$, the magnitude of negative real permeability decays about $2.0 \%$, $28.2 \%$, and $15.1 \%$ at wavelengths of 476,568 , and $676 \mathrm{~nm}$, respectively. However, the magnitude of the negative real part of the permeability obviously decays when the thickness increases from 450 to $480 \mathrm{~nm}$; in addition, the value drops about $18.2 \%, 33.1 \%$, and $41.5 \%$ at wavelengths of 476,568 , and $676 \mathrm{~nm}$, respectively.

Reversal of the magnetic field in the SWF is induced by coupling in the dielectric layer of the antiphase electric fields that oscillate in the top and bottom nanodisks. Also, the magnitude of the negative real part of the permeability is proportional to that of the magnetic dipole moment. The magnetic response of individual sandwiches is simulated by the FDTD method for a sandwich model. The sandwich model consists of coaxial Al nanodisks, separated by a cylindrical $\mathrm{SiO}_{2}$ spacer with a thickness of $45 \mathrm{~nm}$. Since the diameter of the deposited Al nanopillar is a Gaussian distribution judged from the top-view SEM image, the diameter of Al nanodisk for simulation is varied over a range from 100 to $220 \mathrm{~nm}$. The thickness of $\mathrm{Al}$ nanodisk is adopted, as determined by the average thicknesses of the three samples. Figure 6 plots the reversed magnetic field along the $y$-axis at the center of the spacer versus wavelength from 400 to $700 \mathrm{~nm}$ and the diameter of SWF ranging from 100 to $220 \mathrm{~nm}$. Simulation results indicate that the reversal of the magnetic field occurs over the visible wavelengths when the diameter exceeds $160 \mathrm{~nm}$. In the simulation of SWF-A, magnitude of the reversed magnetic field is the largest at a wavelength of $700 \mathrm{~nm}$ with the diameter of $180 \mathrm{~nm}$. The other two simulations for SWF-B and SWF-C have the largest reversal magnetic fields at a wavelength of 642 and $681 \mathrm{~nm}$, respectively. In the three
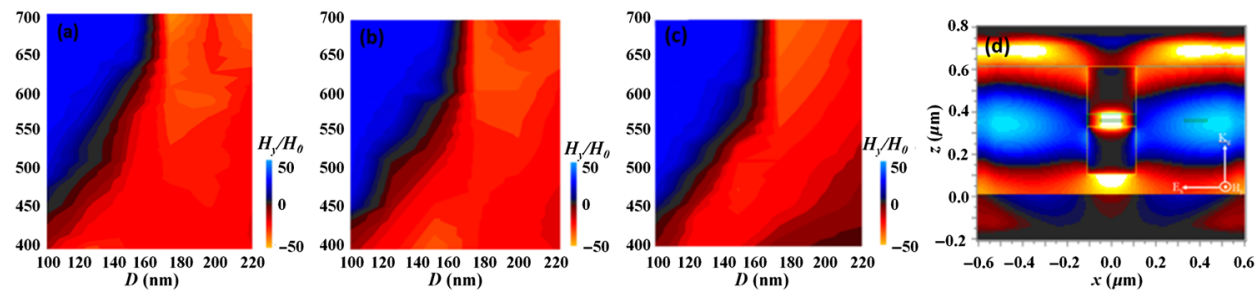

Fig. 6 Simulated intensity of reversed magnetic field $H_{y}$ as a function wavelength (vertical coordinate) and sandwich diameter (i.e., transverse coordinate) for (a) SWF-A, (b) SWF-B, and (c) SWF-C. Thickness of $\mathrm{SiO}_{2}$ film is $45 \mathrm{~nm}$. (d) The inverse magnetic field $H_{y}$ in middle layer of SWF oscillating in a manner opposite to that of the applied magnetic field (blue region). 
Jen, Liao, and Lin: Effect of size of aluminum/silicon dioxide/aluminum nanosandwich films...

simulations, the average magnitude and area of the reversed magnetic field gradually decay as the thickness of SWF increases. Especially for the simulation of SWF-C, the area of the reversed magnetic field obviously shrinks obviously. Results of the FDTD simulation correlate with those of the detected spectra of permeability.

\section{Conclusion}

SWFs of various sizes are fabricated, along with their transmittance, reflectance, and absorptance compared as well. Although the absorptance increases with the SWF thickness, three samples with thicknesses of 420,450 , and $480 \mathrm{~nm}$ exhibit reflectance levels below $1 \%$. The retrieved equivalent refractive index, forward impedance, and backward impedance reveal the propagation of the wave through SWF. Destructive interference for antireflection occurs even when the thickness of SWF is $480 \mathrm{~nm}$. The dispersion of refractive index causes the low dispersion of optical thickness, resulting in destructive interference over the visible wavelengths. The decrease in measured negative equivalent permeability along with the thickness is interpreted qualitatively by simulating the reversed magnetic field at the center of each SWF. Simulation results indicate that the negative permeability arises from the reversal of the magnetic resonance within the $\mathrm{SiO}_{2}$ layer. Furthermore, the reversed magnetic field becomes weaker as the thickness of $\mathrm{Al}$ increases, which corresponds to the trend of measured negative real part of the equivalent permeability.

\section{Acknowledgments}

The authors would like to thank the National Science Council of the Republic of China, Taiwan, for financially supporting this research under Contract Nos. NSC 99-2221-E-027-043-MY3 and NSC 102-2221-E-027-096-MY3.

\section{References}

1. V. M. Shalaev et al., "Negative index of refraction in optical metamaterial," Opt. Lett. 30(24), 3356-3358 (2005), http://dx.doi.org/10.1364/OL.30.003356.

2. A. V. Kildishev et al., "Negative refractive index in optics of metal-dielectric composites," J. Opt. Soc. Am. B 23(3), 423-433 (2006), http://dx.doi.org/10.1364/JOSAB.23.000423.

3. G. Dolling et al., "Simultaneous negative phase and group velocity of light in a metamaterial," Science 312(5775), 892-894 (2006), http://dx.doi.org/10.1126/science.1126021.

4. J. Valentine et al., "Three-dimensional optical metamaterial with a negative refractive index," Nature 455(7211), 376-379 (2008), http://dx.doi.org/10.1038/nature07247.

5. S. Xiao et al., "Yellow-light negative-index metamaterials," Opt. Lett. 34(22), 3478-3480 (2009), http://dx.doi.org/10.1364/OL.34.003478.

6. C. García-Meca et al., "Low-loss multilayered metamaterial exhibiting a negative index of refraction at visible wavelengths," Phys. Rev. Lett. 106(6), 067402 (2011), http://dx.doi.org/ 10.1103/PhysRevLett.106.067402.

7. N. I. Zheludev and Y. S. Kivshar, "From metamaterials to metadevices," Nat. Mater. 11(11), 917-924 (2012), http://dx.doi.org/10.1038/nmat3431.

8. A. V. Kildishev et al., "Negative refractive index in optics of metal-dielectric composites," J. Opt. Soc. Am. B 23(3), 423-433 (2006), http://dx.doi.org/10.1364/JOSAB.23.000423.

9. A. Lakhtakia and R. Messier, Sculptured Thin Films: Nanoengineered Morphology and Optics, SPIE Press, Bellingham, WA (2005), http://dx.doi.org/10.1117/3.585322.ch5.

10. Y.-J. Jen, C.-H. Chen, and C.-W. Yu, "Deposited metamaterial thin film with negative refractive index and permeability in visible regime," Opt. Lett. 36(6), 1014-1016 (2011), http://dx.doi.org/10.1364/OL.36.001014.

11. Y.-J. Jen et al., "Metal/dielectric/metal sandwich film for broadband reflection reduction," Sci. Rep. 3, 1672 (2013), http://dx.doi.org/10.1038/srep01672.

12. C. É. Kriegler et al., "Bianisotropic photonic metamaterials," IEEE J. Sel. Top. Quantum Electron. 16(2), 367-375 (2010), http://dx.doi.org/10.1109/JSTQE.2009.2020809. 
Yi-Jun Jen is professor and chairman of the Department of Electro-optical Engineering in National Taipei University of Technology (NTUT), Taiwan. He received MS and PhD degrees from the Institute of Optical Sciences of National Central University in 1994 and 2001, respectively. He joined the Department of Electro-optical Engineering, NTUT in 2002. His current research interests primarily involve fabrication of novel optical devices based on anisotropic thin films, optical property analysis for subwavelength scale composite material.

Hung-Sheng Liao received the BSc degree in the Department of Electro-optical Engineering at the Minghsin University of Science and Technology, Taipei, Taiwan, in 2009. He received the MSc degree from the Department of Electro-optical Engineering at the National Taipei University of Technology, where he is studying anisotropic optical analysis and nanosculptured thin films.

Meng-Jie Lin received the BSc degree in the Department of Electro-optical Engineering at the National Taipei University of Technology, Taipei, Taiwan, in 2008. He is currently pursuing the MSc degree and PhD degree in the Department of Electro-optical Engineering at the National Taipei University of Technology, where he is studying anisotropic optical analysis and optical property analysis for subwavelength scale composite material. 